\title{
Characterizing saccular aortic arch aneurysms from the geometry-flow dynamics relationship
}

\author{
Kayoko Natsume, MD, ${ }^{\text {a }}$ Norihiko Shiiya, MD, PhD, ${ }^{\mathrm{a}}$ Yasuo Takehara, MD, ${ }^{\mathrm{b}}$ Masataka Sugiyama, MD, ${ }^{\mathrm{b}}$ \\ Hiroshi Satoh, $\mathrm{MD}, \mathrm{PhD},{ }^{\mathrm{c}}$ Katsushi Yamashita, $\mathrm{MD}, \mathrm{PhD},{ }^{\mathrm{a}}$ and Naoki Washiyama, $\mathrm{MD}, \mathrm{PhD}^{\mathrm{a}}$
}

\section{ABSTRACT}

Objective: Low wall shear stress (WSS) has been reported to be associated with accelerated atherosclerosis, aneurysm growth, or rupture. We evaluated the geometry of aortic arch aneurysms and their relationship with WSS by using the 4-dimensional flow magnetic resonance imaging to better characterize the saccular aneurysms.

Methods: We analyzed the geometry in 100 patients using multiplanar reconstruction of computed tomography. We evaluated WSS and vortex flow using 4-dimensional flow magnetic resonance imaging in 16 of them, which were compared with 8 age-matched control subjects and eight healthy young volunteers.

Results: Eighty-two patients had a saccular aneurysm, and 18 had a fusiform aneurysm. External diameter/aneurysm length ratio and sac depth/neck width ratio of the fusiform aneurysms were constant at $0.76 \pm 0.18$ and $0.23 \pm 0.09$, whereas those of saccular aneurysms, especially those involving the outer curvature, were higher and more variable. Vortex flow was always present in the aneurysms, resulting in low WSS. When the sac depth/neck width ratio was less than 0.8, peak WSS correlated inversely with luminal diameter even in the saccular aneurysms. When this ratio exceeded 0.8 , which was the case only with the saccular aneurysms, such correlation no longer existed and WSS was invariably low.

Conclusions: Fusiform aneurysms elongate as they dilate, and WSS is lower as the diameter is larger. Saccular aneurysms dilate without proportionate elongation, and they, especially those occupying the inner curvature, have higher and variable sac depth/neck width ratio. When this ratio exceeds 0.8 , WSS is low regardless of diameter, which may explain their malignant clinical behavior. (J Thorac Cardiovasc Surg 2017;153:1413-20)

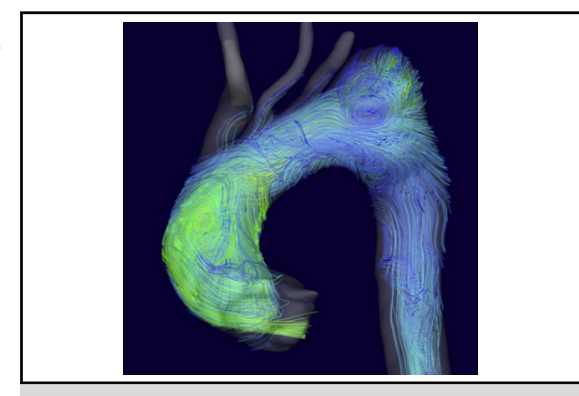

Vortex flow was present in the ascending aorta and aneurysm.

\section{Central Message}

Saccular arch aneurysms dilate without proportionate elongation. They, especially those occupying inner curvature, have low wall shear stress regardless of diameter and may therefore behave malignantly.

\section{Perspective}

Fusiform arch aneurysms elongate as they dilate, and wall shear stress is lower as the diameter is larger. Saccular arch aneurysms dilate without proportionate elongation, and they, especially those occupying the inner curvature, have higher and variable sac depth/ neck width ratio. When this ratio exceeds 0.8 , wall shear stress is low regardless of diameter, which may explain their malignant behavior.

See Editorial Commentary page 1421.

See Editorial page 1411.
Saccular aortic aneurysms generally are considered to have an elevated risk of rupture compared with fusiform ones; however, little is known about the influence of geometry and size of the saccular aneurysms on their risk. Using finite element analysis (FEA) of the simulated abdominal aortic

\footnotetext{
From the ${ }^{\mathrm{a}}$ First Department of Surgery, Departments of ${ }^{\mathrm{b}}$ Radiology, and ${ }^{\mathrm{c}}$ Internal Medicine III, Hamamatsu University School of Medicine, Shizuoka, Japan.

Received for publication Aug 12, 2016; revisions received Oct 26, 2016; accepted for publication Nov 4, 2016; available ahead of print Dec 24, 2016.

Address for reprints: Kayoko Natsume, MD, 1-20-1 Handayama, Higashi-ku, Hamamatsu, Shizuoka 431-3192, Japan (E-mail: D14022@hama-med.ac.jp). $0022-5223 / \$ 36.00$

Copyright (c) 2016 by The American Association for Thoracic Surgery http://dx.doi.org/10.1016/j.jtcvs.2016.11.032
}

aneurysms (AAAs) that had more or less asymmetric dilatation, Vorp and colleagues ${ }^{1}$ reported that both diameter and shape affected the wall stress in AAA. Nathan and colleagues $^{2}$ reported that diameter-normalized wall stress assessed by FEA for saccular descending aortic aneurysms was greater than that for fusiform aneurysms. These reports, however, did not take account of the presence of mural

Scanning this $\mathrm{QR}$ code will take you to supplemental table and video for this article.

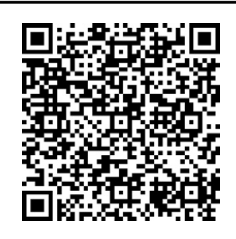




$$
\begin{aligned}
& \text { Abbreviations and Acronyms } \\
& \text { AAA }=\text { abdominal aortic aneurysms } \\
& \text { CFD }=\text { computational fluid dynamics } \\
& \text { CT }=\text { computed tomography } \\
& \text { FEA }=\text { finite element analysis } \\
& \text { 4D }=4 \text { dimensional } \\
& \text { MRI }=\text { magnetic resonance imaging } \\
& \text { OSI }=\text { oscillatory shear index } \\
& \text { WSS }=\text { wall shear stress }
\end{aligned}
$$

thrombus or calcification. Shang and colleagues ${ }^{3}$ reported that diameter-normalized wall stress calculated by FEA was not different between saccular and fusiform descending aortic aneurysms when mural thrombus was taken into account as wall thickness. These reports make us wonder what shape of aneurysm is associated with elevated risk of rupture and what the clinically relevant definition of the saccular aneurysm from this point of view is.

In the distal aortic arch and proximal descending aorta, we encounter saccular aortic aneurysms more frequently than in other areas. Shang and colleagues ${ }^{4}$ reported that saccular aortic aneurysms comprised only $1.5 \%$ of the entire aortic aneurysms. The geometry of aortic arch is characterized by curvature and presence of major branches on the outer curvature. Therefore, we guess that such characteristics play an important role in the development and natural course of saccular aortic aneurysms in this curved region.

Four-dimensional (4D) flow analysis that is derived from the measured data of the magnetic resonance imaging (MRI) and computational fluid dynamics (CFD) simulation recently has been reported to evaluate flow dynamics of the aorta $^{5}$ or cerebral aneurysms, and effect of aortic valve configuration. ${ }^{6,7}$ Concerning the cerebral aneurysms, these methods have been used to elucidate the relationship between aneurysm geometry and the risk of growth and rupture, and parameters derived from them, such as wall shear stress (WSS) and oscillatory shear index (OSI), has been reported to be associated with rupture. ${ }^{8-10}$ In the AAA, Chisci and colleagues ${ }^{11}$ reported the usefulness of CFD-derived OSI to predict the risk of rupture. In the thoracic aorta, however, reports of such an analysis have been sparse. ${ }^{12,13}$

We evaluated the geometry of both saccular and fusiform aortic aneurysms in the aortic arch curvature from the multiplanar reconstruction of the contrast-enhanced computed tomography (CT) data and its relationship with WSS and OSI within them from the $4 \mathrm{D}$-flow MRI. The objective was to determine the geometry and location, in relation to the aortic curvature, of the saccular aortic arch aneurysms that had these parameters distinct from those of fusiform aneurysms, so that we can better characterize them to stratify their risk.

\section{MATERIALS AND METHODS} Aneurysm Geometry Study

We retrospectively analyzed the geometry of aneurysms involving the distal aortic arch to proximal descending aorta in 100 patients who underwent contrast-enhanced CT between April 2009 and December 2015 at our institution. Patients' ages were $75 \pm 7$ years, and $64 \%$ were male. All were scheduled to undergo surgical treatment, and CT was a preoperative examination. Patients with multiple aneurysms in this region, aortic dissection, aortitis, infected aneurysms, connective tissue disorder including Marfan syndrome, dilated ascending aorta $(>40 \mathrm{~mm})$, and history of previous surgery on the ascending aorta to aortic arch were excluded from the analysis. This study was approved by the institutional review board (16-012).

To analyze the geometry of aneurysms, we used a modeling workstation (SYNAPSE VINCENT, Fujifilm medical, Tokyo, Japan) to process the CT data. As parameters of aneurysm geometry, external diameter, luminal diameter, aneurysm length, and neck width were measured from multiplanar reconstruction images, together with the luminal diameters of proximal and distal aorta (Figure 1). An average of proximal and distal luminal diameter was used as a luminal diameter of the nonaneurysmal aorta. Sac depth was calculated as a difference of luminal diameter between the aneurysm and nonaneurysmal aorta.

Patients were divided into the following 3 groups according to the geometry and location of the aneurysms; saccular aneurysms located on the inner curvature or ventral surface of aortic arch (saccular inner group), those located on outer curvature of aortic arch (saccular outer group), and fusiform aneurysms (fusiform group). Aneurysms were considered saccular when aortic dilation was focal and asymmetric with a portion of aortic circumference considered normal. ${ }^{14}$

\section{D-Flow MRI Study}

Study subjects consisted of 16 patients who had aneurysms involving the distal aortic arch to proximal descending aorta and 8 young healthy volunteers and age-matched control subjects without aortic disease who underwent 4D-flow MRI for some reasons related to the underlying disease. Subjects having aneurysms also were included in the aneurysm geometry study. All participants underwent 4D-flow MRI between November 2011 and October 2015 at our institution. A 3-Tesla magnetic resonance scanner (Discovery MR750 or MR750w; GE Healthcare, Waukesha, Wis) was used. All participants had a tricuspid aortic valve. Patients with an aortic valve disease or ejection fraction less than $60 \%$ on transthoracic echocardiography were not included. This study was approved by the institutional review board (16-012, 22-45, 25-236), the geometry study was approved by the institutional review board (16-012), and the 4D-flow study was approved by the institutional review board (22-45, 25-136); a written informed consent was obtained from all subjects.

4D-flow imaging. Contrast-enhanced 3D magnetic resonance angiography was performed first to create a shape of aortic wall. Bolus injection of $0.1 \mathrm{mmol} / \mathrm{kg}$ gadolinium chelate (Omniscan; Daiichi Pharma Co., Japan) was performed at an injection rate of $2.0 \mathrm{~mL} / \mathrm{s}$ followed by $20 \mathrm{~mL}$ of saline at the same injection rate. For 4D-flow imaging, respiration compensated retrospective cardiac gating was used. Raw data were transferred to a personal computer for postprocessing and flow visualization in a DICOM (ie, Digital Imaging and Communications in Medicine) format. A flow analysis software Flova ( $\mathrm{R}^{\prime}$-Tech Co, Hamamatsu, Japan) was used to visualize intraaortic flow information with the spatial resolution of $2 \times 2 \times 2 \mathrm{~mm}$.

Analysis of flow pattern. Flow patterns in the ascending aorta and in the aneurysms were evaluated visually from the streamlines and particle traces for the presence of vortex flow (Figure 2 and Video 1).

Analysis of flow dynamics. For calculation of the parameters of flow dynamics, we manually set 6 cross-sectional planes; namely, immediately above the sino-tubular junction (plane 1), just proximal to the origin of brachiocephalic trunk (plane 3), ascending aorta at the half way between the plane 1 and 3 (plane 2), just proximal to the origin of left common carotid artery (plane 4), just distal to the origin of left common carotid artery 

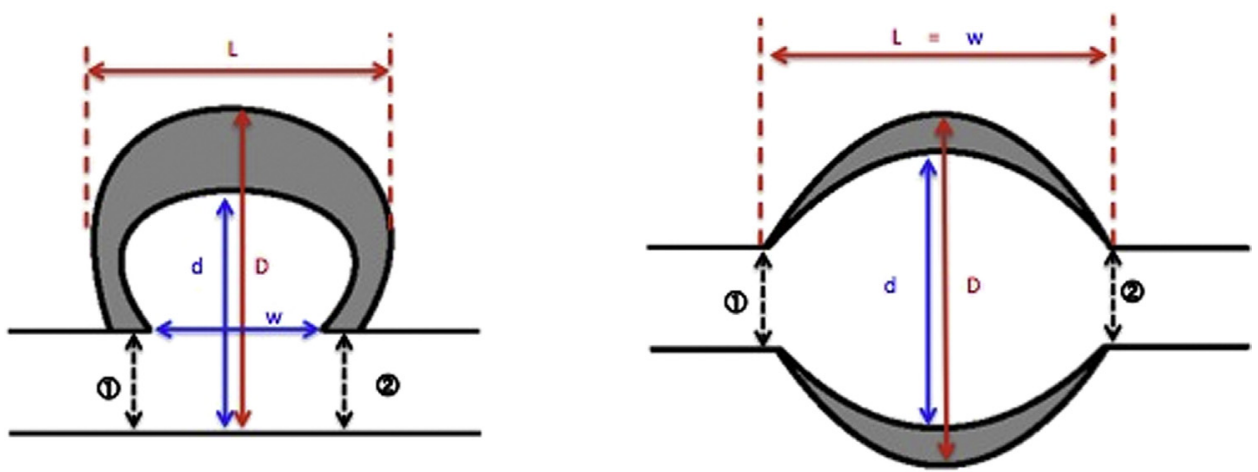

FIGURE 1. Definition of aneurysm geometry. Left, saccular aneurysms, Right, fusiform aneurysms. Luminal diameter of the nonaneurysmal aorta was calculated as $(1)+(2) / 2$. $L$, Aneurysm length; $d$, luminal diameter; $D$, external diameter; $w$, neck width.

(plane 5), and proximal descending aorta (plane 6) (Figure 3). All planes were placed perpendicular to the longitudinal vessel axis. We regarded the average of planes 1 to 3 as the ascending aorta and that of planes 4 to 6 as the aortic arch for calculation of WSS and OSI in these areas. In the patients with aneurysms, other planes were set in the proximal, middle, and distal end of the aneurysm.
As parameters of flow dynamics, net flow velocity through each plane and net WSS and OSI of the circumferential luminal surface of each plane were calculated (Appendix 1). Change in the net flow velocity $(\mathrm{m} / \mathrm{s})$ during one cardiac cycle was measured in 20 phases and was averaged. A 3D method reported by Isoda and colleagues ${ }^{15}$ was used for WSS calculation from MRI data. OSI, a mean fluctuation of WSS vectors during one cardiac

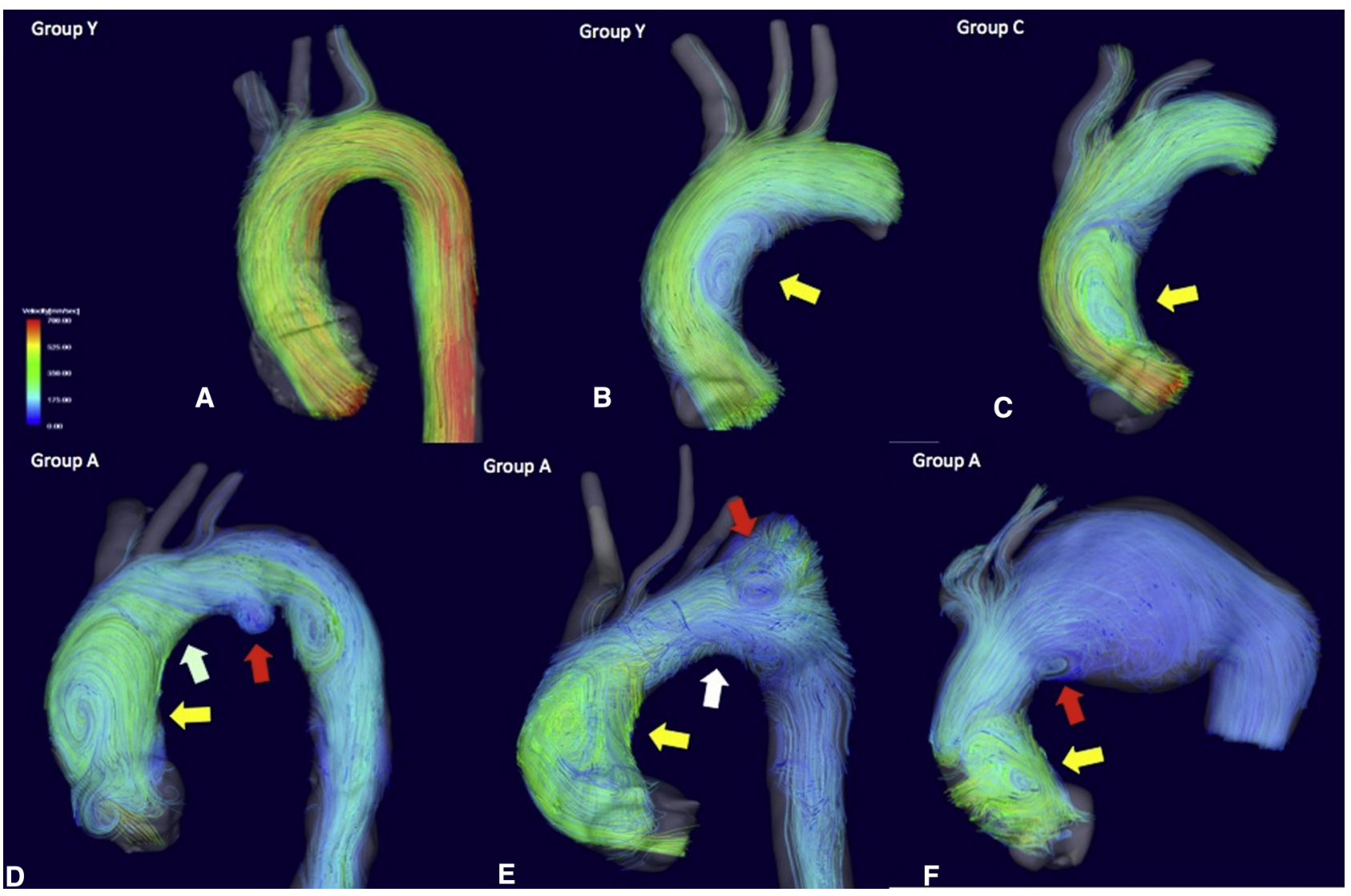

FIGURE 2. Representative cases of 3-dimensional streamline visualization during systole. Case A: A young healthy volunteer. Flow was laminar in the ascending aorta. Case B: A young healthy volunteer. Vortex flow (yellow arrow) was present in the ascending aorta. Case C: An age-matched control subject. Vortex flow (yellow arrow) was present in the ascending aorta. Case D: A patient with a saccular inner type aneurysm. Vortex flow was present in the ascending aorta (yellow arrow), and helix flow was present in the aortic arch (green arrow). The vortex flow occupied the entire aneurysm (red arrow). Case E: A patient with a saccular outer type aneurysm. Vortex flow was present in the ascending aorta (yellow arrow) and within the aneurysm (red arrow), and turbulent flow was present in the aortic arch (white arrow). Case F: A patient with a fusiform aneurysm. Vortex flow was present in the ascending aorta (yellow arrow) and within the aneurysm (red arrow). Group Y, Young healthy volunteer; Group C, age-matched control; Group A, patients with an aneurysm. 


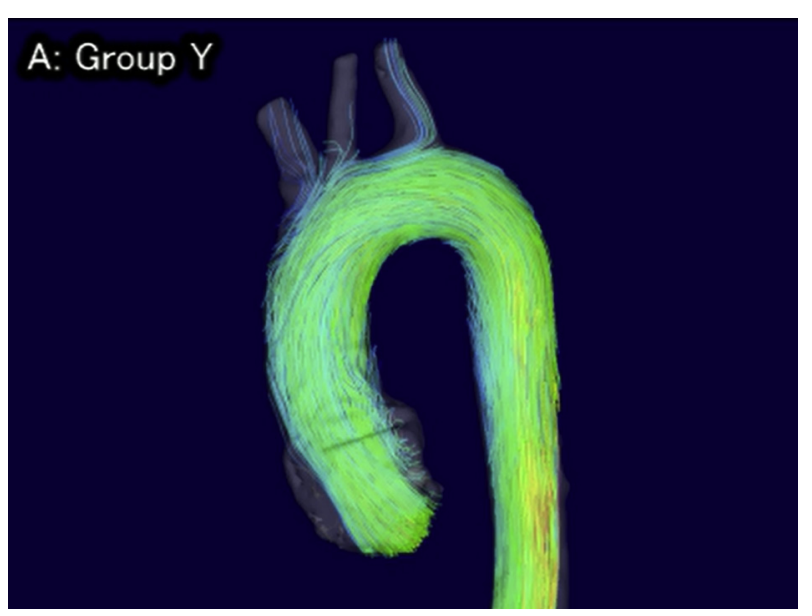

VIDEO 1. Representative cases of 3-dimensional streamline visualization. Case A: A young healthy volunteer. Flow was laminar in the ascending aorta. Case B: A young healthy volunteer. Vortex flow was present in the ascending aorta. Case C: An age-matched control subject. Vortex flow was present in the ascending aorta. Case D: A patient with a saccular inner type aneurysm. Vortex flow was present in the ascending aorta, and helix flow was present in the aortic arch. The vortex flow occupied the entire aneurysm. Case E: A patient with a saccular outer type aneurysm. Vortex flow was present in the ascending aorta and within the aneurysm, and turbulent flow was present in the aortic arch. Case F: A patient with a fusiform aneurysm. Vortex flow was present in the ascending aorta and within the aneurysm. Group Y, Young healthy volunteer; Group $C$, age-matched control; Group A, patients with an aneurysm. Video available at: http://www. jtcvsonline.org/article/S0022-5223(16)31626-9/addons.

cycle, ranges from 0 to $0.5 .{ }^{16}$ Large OSI implies that WSS vectors fluctuate greatly.

\section{Statistical Analysis}

An IBM SPSS Statistics 22 software (IBM Inc, Armonk, NY) was used for all statistical analyses. The Pearson $\chi^{2}$ test was used for comparison of nominal variables and 1-way analysis of variance test was used for continuous variables. The Levene test was used to evaluate the equality of variances. As post-hoc tests, we used Bonferroni procedure when variances were equal or Games-Howell procedure when they were not. Correlation between the continuous variables was evaluated by Pearson rank correlation coefficient. Influence of 2 factors on one continuous variable was evaluated by 2 -way analysis of variance test. $P<.05$ was considered significant. All results were presented as mean \pm standard deviation.

\section{RESULTS}

\section{Aneurysm Geometry Study}

Eighty-two patients $(82 \%)$ had a saccular aneurysm with the predominance of inner location $(\mathrm{n}=59,59 \%)$, and only $18(18 \%)$ had a fusiform aneurysm. External diameter was $59 \pm 12 \mathrm{~mm}$, with no difference among the 3 groups. Luminal diameter was $46 \pm 10 \mathrm{~mm}$, and patients in the fusiform group had significantly larger luminal diameter $(54 \pm 10 \mathrm{~mm})$ than those in the saccular inner group (45 $\pm 9 \mathrm{~mm})$ and saccular outer group $(42 \pm 7 \mathrm{~mm})(P<.001, P<.001)$. External diameter/aneurysm length ratio, sac depth/neck width ratio, and their variance were largest in the saccular inner group, followed by the saccular outer group (Figure 4). There was a significant correlation between the external diameter/aneurysm length ratio and sac depth/neck width ratio $(r=0.587$, $P<.001)$. Of note, the external diameter/aneurysm length ratio and sac depth/neck width ratio were fairly constant at $0.76 \pm 0.18$ and $0.23 \pm 0.09$ in the fusiform group.

\section{D-Flow MRI Study}

Patient characteristics. Mean age (year) of the 16 aneurysm patients was $74 \pm 6.9$, and those of 8 age-matched control subjects and 8 healthy young volunteer were $70 \pm 6.9$ and $40 \pm 9.9$, respectively. Patients with aneurysms belonged to the saccular inner group in 7, the saccular outer group in 5, and the fusiform group in 4 in the aneurysm geometry study. There was a significant difference in the diameter of ascending aorta $(34 \pm 3.6 / 32 \pm 3.0 / 26 \pm 1.2 \mathrm{~mm}, P<.001)$ and aortic arch $(33 \pm 2.8 / 30 \pm 2.224 \pm 1.1 / \mathrm{mm}, P<.001)$ among the patients with aneurysms, age-matched control subjects, and young healthy volunteers.

Flow pattern (Figure 2, Video 1). In the ascending aorta, vortex flow rarely was observed in the young healthy volunteers, whereas large vortex flow was almost always present in the patients with aneurysms and age-matched control subjects, who had larger aortic diameter. In the aortic arch, all subjects in the young healthy volunteer showed laminar flow, whereas age-matched control subjects and patients with aneurysms showed helical or turbulent flow. In the patients with aneurysms, all patients showed vortex flow within the aneurysm.

Flow dynamics. Change in the net flow velocity through each plane during one cardiac cycle is depicted in Figure 3. In all planes, peak flow velocity was significantly different among the 3 groups. In plane 1 of the patients with aneurysms, net systolic velocity was bimodal because of the vortex flow.

Figure 5 shows 3D mapping of the peak WSS and OSI. WSS and OSI data were summarized in Table E1. In the ascending aorta and nonaneurysmal aortic arch, peak WSS were the greatest in young healthy volunteers, followed by age-matched control subjects. It was the lowest in patients having aneurysms. In contrast, OSI was not significantly different except for the difference in the ascending aorta between young healthy volunteer and age-matched control subjects $(P=.034)$. In the ascending aorta, significant correlation was found between peak WSS and diameter $(r=-0.763, P<.001)$. In the patients with aneurysms, peak and average WSS and OSI within the aneurysms were significantly lower than those in the non-aneurysmal aortic arch $(P=.001,<.001, .003$, respectively). There was no significant difference among the 3 aneurysm subgroups (saccular inner, saccular outer, fusiform). Even when the influences of luminal diameter 

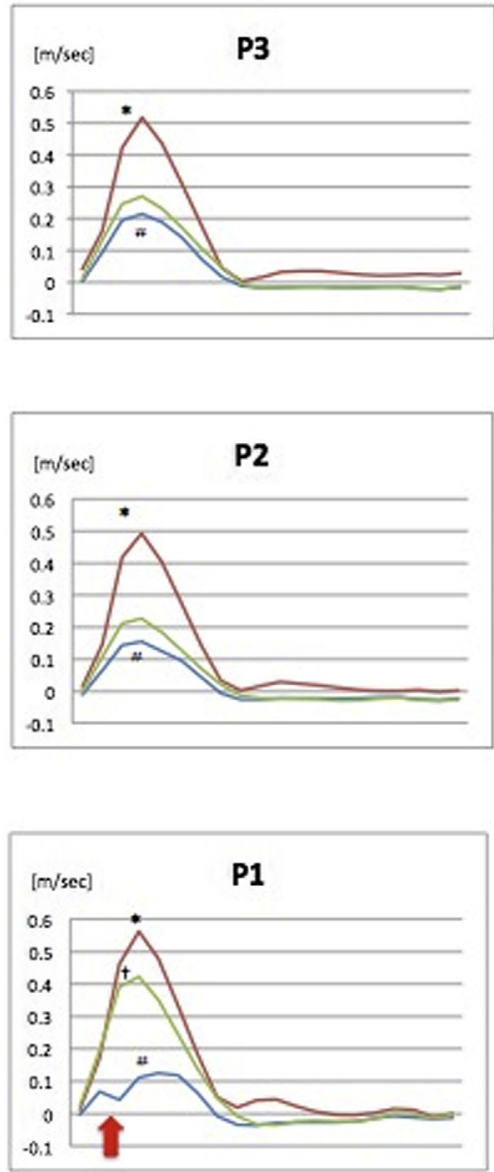

Velocity average $[\mathrm{m} / \mathrm{sec}]$
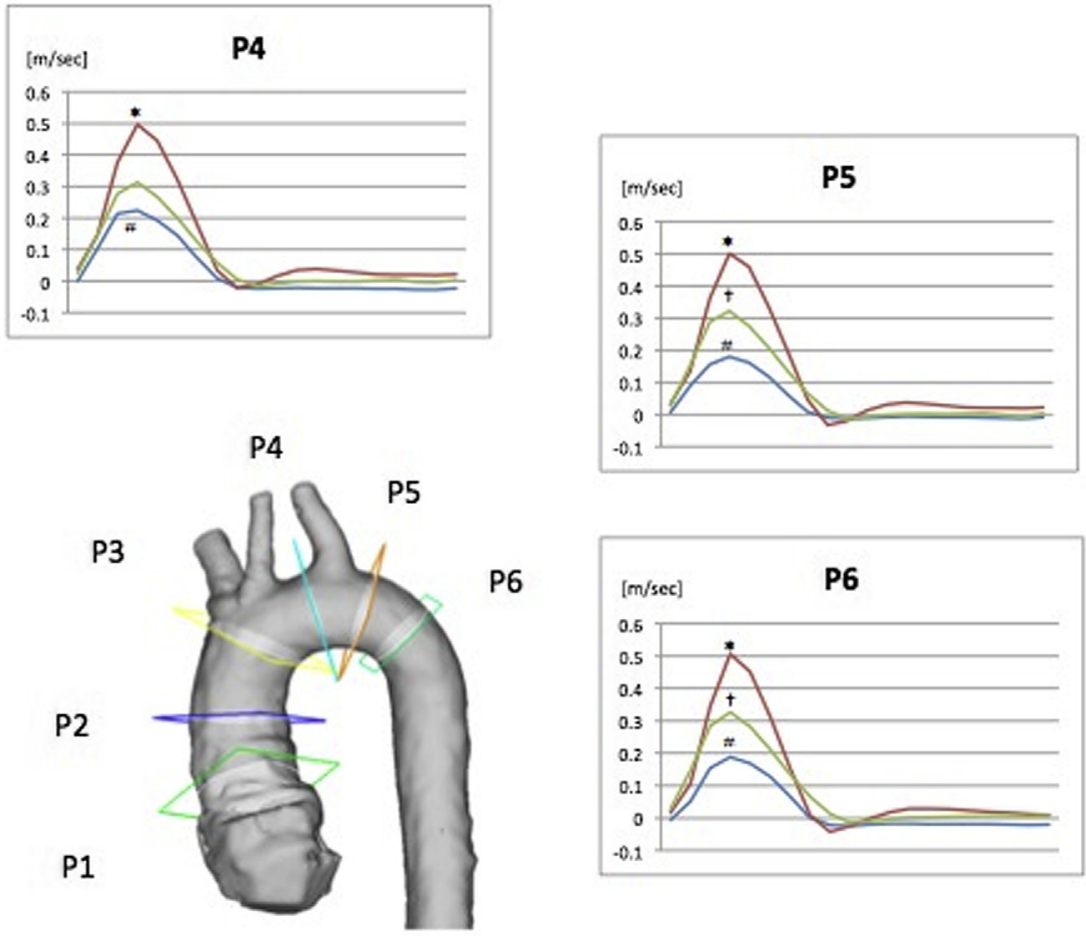

FIGURE 3. Setting of 6 cross-sectional planes (P1-6) and change in the net flow velocity during one cardiac cycle in each plane. In the plane 1 of the group $\mathrm{A}$, net systolic velocity was bimodal (red arrow). $* P<.05$, group Y versus $\mathrm{C}, \dagger P<.05$, group $\mathrm{C}$ versus $\mathrm{A}, \# P<.05$, group Y versus A. Group $A$, Patients with an aneurysm; Group $Y$, young healthy volunteer; Group $C$, age-matched control.

and external diameter on these parameters were analyzed, no significant correlation was found. In contrast, there was a significant inverse correlation between sac depth/neck width ratio and peak WSS $(\mathrm{r}=-0.587$, $P=.017$ ). When sac depth/neck width ratio was less than 0.8 , there was a significant inverse correlation between luminal diameter and peak WSS $(\mathrm{r}=-0.740, P=.006)$ and external diameter and peak WSS $(\mathrm{r}=-0.585$, $P=.046$ ) (Figure 6). Two-way analysis of variance test revealed that interaction between sac depth/neck width ratio $>0.8$ and luminal diameter $<50 \mathrm{~mm}$ had significant influence on peak WSS $(P=.012)$.

\section{DISCUSSION}

The present study showed that the external diameter/ aneurysm length ratio and sac depth/neck width ratio of the fusiform aneurysms were fairly constant, whereas those of saccular aneurysms were higher and more variable. This finding suggests that dilatation of the fusiform aneurysms is accompanied by longitudinal extension, whereas that of the saccular aneurysms is not. It is natural that the external diameter/aneurysm length ratio correlated with sac depth/ neck width ratio. The external diameter/aneurysm length ratio and sac depth/neck width ratio of the saccular aneurysms occupying the outer curvature was smaller than that of those located in the inner curvature, possibly because the outer curvature is naturally longer than the inner curvature.

Such a difference in geometry had a significant influence upon the flow dynamics. Generally, peak WSS became lower as the sac depth/neck width ratio became larger. ${ }^{17}$ When the sac depth/neck width ratio was less than 0.8 , peak WSS showed significant inverse correlation with 

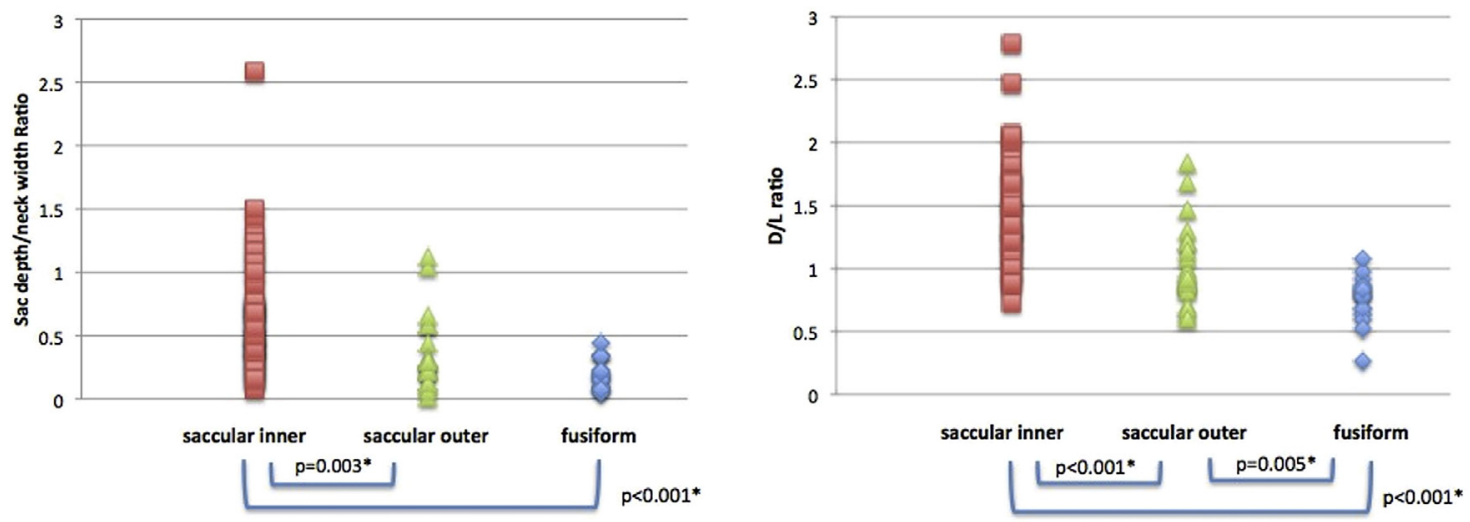

FIGURE 4. Sac depth/neck width ratio, external diameter/aneurysm length ratio according to the three aneurysm types. $D$, External diameter; $L$, aneurysm length.

luminal diameter even in the saccular aneurysms. Consequently, peak WSS was always less than 0.25 when the luminal diameter exceeded $50 \mathrm{~mm}$, or when the sac depth/neck width ratio exceeded 0.8 regardless of luminal diameter, which was the case only with the saccular aneurysms.

These results may suggest that flow dynamics of the saccular aneurysms is different from that of the fusiform ones when the sac depth/neck width ratio is higher than 0.8 , and saccular aneurysms involving the outer curvature may behave more like fusiform ones than those involving the inner curvature from the standpoint of flow dynamics. In the intracranial aneurysms, it has been shown that WSS has inverse correlation with the sac depth/neck width ratio and WSS drastically decreases at sac depth/neck width ratio of 1.5 1.6. ${ }^{17,18}$ Our results suggest that, in the aortic arch curvature, this threshold value seems around 0.8 .

Another important finding of the geometry study was the prevalence of saccular aneurysms, which represented $82 \%$ of the aneurysms involving the distal aortic arch and proximal descending aorta. This was in clear contrast to the report by Shang and colleagues ${ }^{4}$ and may be explained by racial difference or unclear definition of the saccular aneurysms in their study.

In the flow pattern analysis of the ascending aorta, vortex flow was larger as the aortic diameter became larger. This relationship enhanced the correlation between peak WSS and aortic diameter, because the presence of large vortex flow itself resulted in low peak WSS. WSS is calculated from vessel radius, flow volume and blood viscosity, so that these relationships are logical. Other factors such as the shape of aorta may affect WSS in addition to the aortic diameter. Burk and colleagues ${ }^{12}$ reported that presence of vortex flow was associated with both ascending aortic diameter and the ratio of ascending/descending aortic diameter. We also examined the relationship with this ratio, in addition to the length and angle of the ascending aorta, but could not find significant correlation (data not shown).
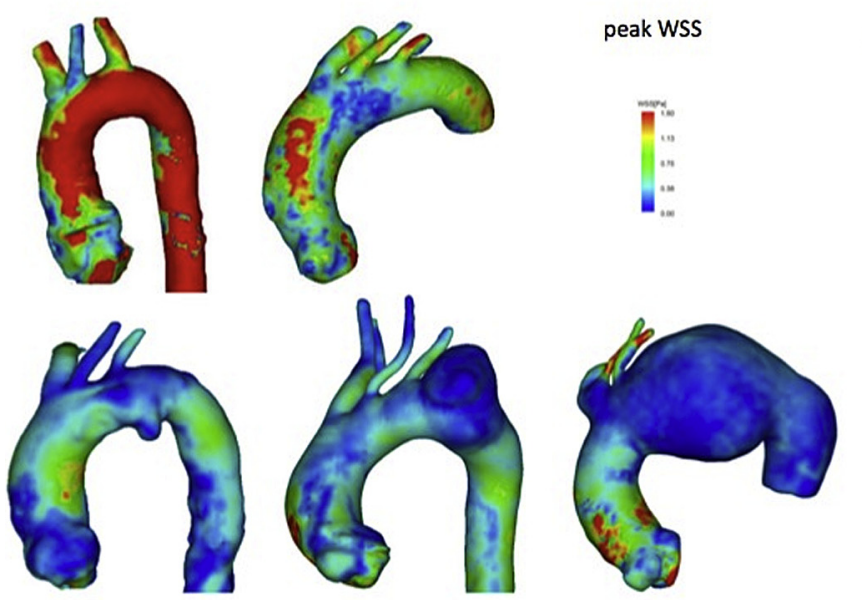
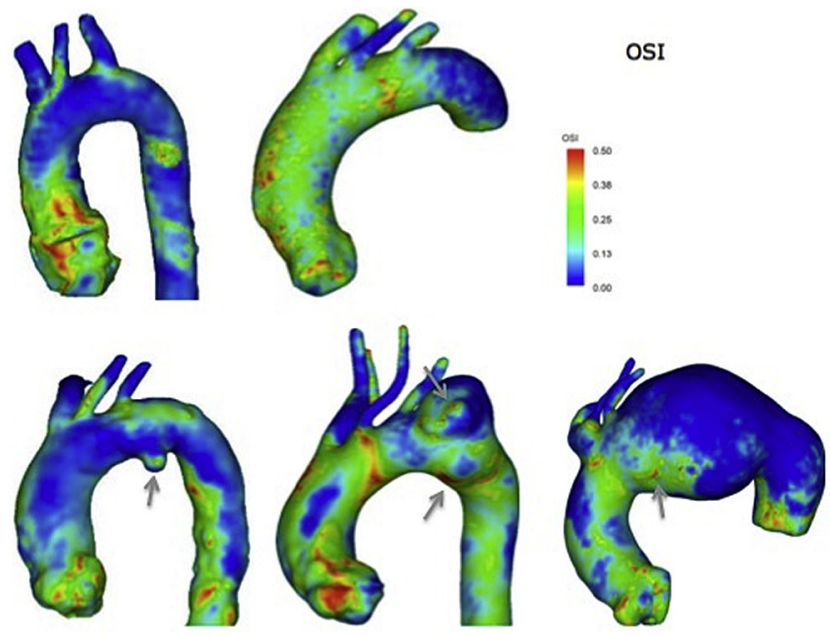

FIGURE 5. Three-dimensional mapping of the peak WSS and OSI. Arrow indicates high OSI area. WSS, Wall shear stress; OSI, oscillatory shear index. 

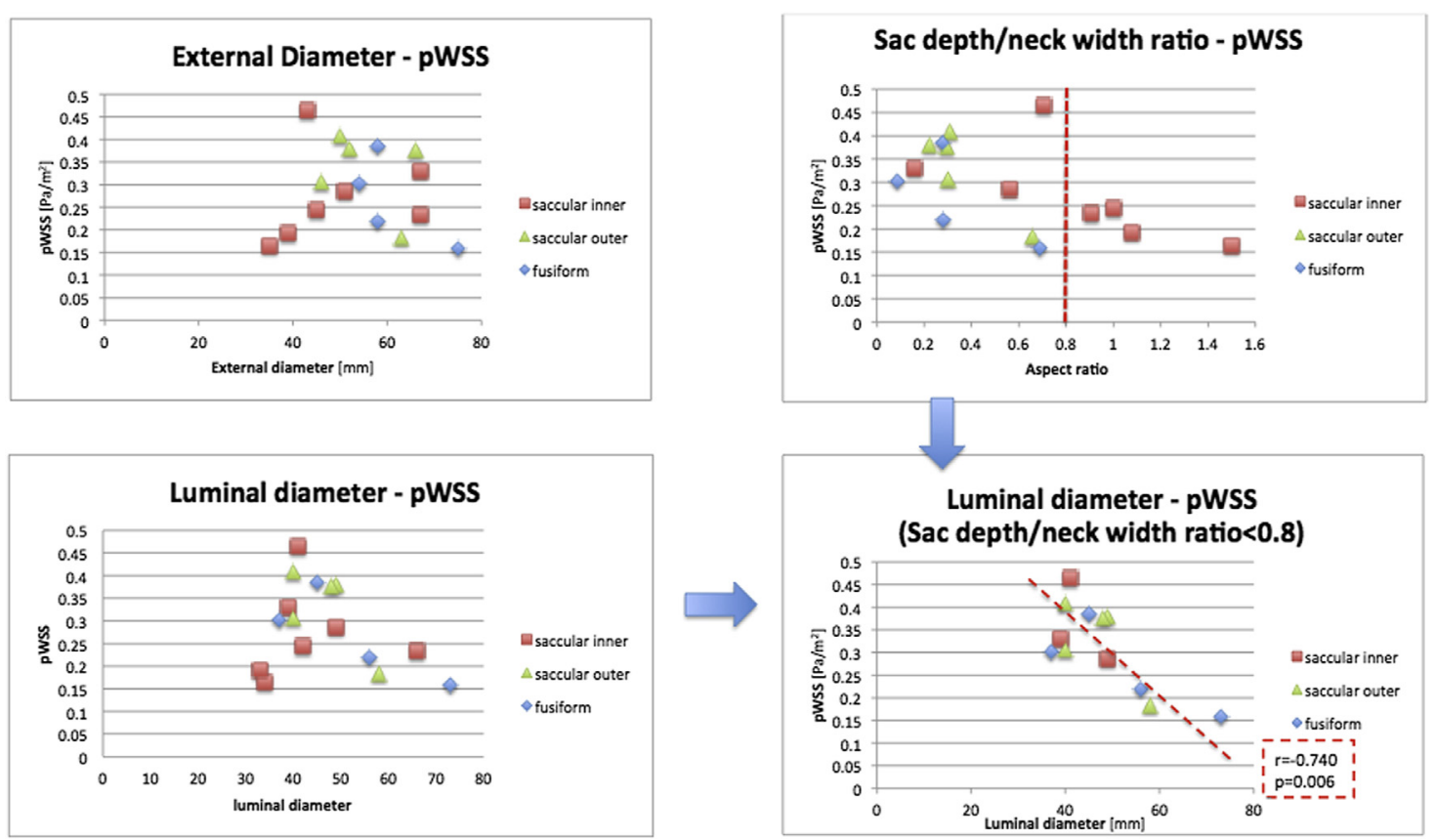

FIGURE 6. Correlation between pWSS and external diameter, luminal diameter, and sac depth/neck width ratio. $p$ WSS, Peak wall shear stress.

High OSI has been reported to be a critical wall parameter indicative of vascular remodeling, aneurysm progression, and risk of rupture. ${ }^{8,10,12,19,20}$ Although we could not find a clear relationship between OSI and aortic diameter or presence of aneurysms, high OSI area was small and its distribution was scattered, so that comparison of the average value may not be meaningful. Because OSI is a mean fluctuation of WSS vectors, it becomes high where blood flow detaches from the main flow to form a vortex or turbulent flow, such as the aneurysm neck. High OSI area is not necessarily present at the largest portion of aneurysms, which is consistent with the work of Boyd and colleagues ${ }^{21}$ showing that rupture site of AAA is not necessarily at the largest portion of aneurysms. These reports suggest that not only the wall tension that is calculated from the Laplace's low but also the parameters of flow dynamics are important to understand the clinical behavior of the aneurysms.

\section{Clinical Implications}

It has been shown that low WSS initiate and exacerbate atherosclerosis through its effects on the endothelial cell function. The adequate level of WSS $\left(>1.5 \mathrm{~Pa} / \mathrm{m}^{2}\right)$ keeps it atheroprotective, whereas low WSS $\left(<0.4 \mathrm{~Pa} / \mathrm{m}^{2}\right)$ switches the endothelial phenotype to atherogenic. ${ }^{19}$ Within the aneurysms, low-velocity vortex flow always was present and, as a consequence, WSS decreased. The pathophysiology of aortic aneurysms is characterized by inflammatory cell infiltration and destruction/reconstruction of the wall structure with complicated atherosclerosis. ${ }^{22}$ The extracellular matrix of aortic wall is degraded by activation of matrix metalloproteinase. Cheng and colleagues ${ }^{23}$ have reported that great activity of matrix metalloproteinase was observed in the low WSS area. Taken together, the low WSS within the aneurysms may contribute to weakening of vessel wall and promoting aneurysm growth. This means that once an aneurysm is formed, WSS becomes low, especially so in the saccular aneurysms, which promotes aneurysm growth, thereby forming a vicious cycle.

In the intracranial aneurysms, Jing and colleagues ${ }^{9}$ have reported that ruptured aneurysms have lower WSS and higher OSI compared with unruptured aneurysms. Boussel and colleagues ${ }^{8}$ have demonstrated the relationship between local aneurysm growth and areas of low WSS in untreated intracranial aneurysms. In the AAA, Boyd and colleagues $^{21}$ have reported that rupture occurred at the site of flow recirculation and low WSS. Although we did not have data on the aneurysm growth or rupture, these reports suggest that low WSS within the aneurysms is associated with an increased risk of rupture.

4D-flow MRI is costly and time-consuming, which makes its application to the patients unrealistic in our daily practice. On the other hand, the sac depth/neck width ratio can readily be calculated from a contrast enhanced CT data. For the patients with saccular aortic arch aneurysms, this ratio may be used to estimate the risk. When this ratio is less than 0.8 , the size criteria may be appropriate in decision making. When it is more than 0.8 , it is advisable to consider that the risk of aneurysm rupture is high regardless of its diameter. 


\section{Study Limitations}

This is a retrospective study in a single institution with its inherent bias. The sample size for 4D-flow MRI study was small. 4D-flow MRI uses blood flow velocity data and aortic luminal shape from MRA, so the obtained parameters do not reflect the aortic wall characteristics, such as presence of thrombus or calcification. Patients were evaluated at a single time point before surgery, and no information on the growth rate or the rupture site was available. Histopathology of the aortic wall also was not available. Therefore, we cannot draw any conclusions on the causal relationship between flow dynamics and aneurysm formation or their prognosis. MRI cannot be applied in the emergency situation. In this regard, CFD may be useful, but we believe that direct measurements by $4 \mathrm{D}$-flow are superior to simulation in the elective situation.

\section{CONCLUSIONS}

Dilatation of the fusiform aneurysms is accompanied by longitudinal extension, whereas that of the saccular aneurysms is not, which results in higher and more variable sac depth/neck width ratio in the latter, especially those occupying the inner curvature. When this ratio is higher than 0.8 , a situation only seen with the saccular aneurysms, WSS is invariably low, whereas WSS inversely correlates with the luminal diameter when it is less than 0.8. Because low WSS has been reported to exacerbate atherosclerosis and to be associated with aneurysm growth or rupture, these results suggest that the clinical behavior of small saccular aortic arch aneurysms may become worse when their sac depth/neck width ratio exceeds 0.8 , and those occupying the inner curvature may generally be more malignant than those occupying the outer curvature.

\section{Conflict of Interest Statement}

Authors have nothing to disclose with regard to commercial support.

\section{References}

1. Vorp DA, Raghavan ML, Webster MW. Mechanical wall stress in abdominal aortic aneurysm: influence of diameter and asymmetry. J Vasc Surg. 1998;27: 632-9.

2. Nathan DP, Xu C, Pouch AM, Chandran KB, Desjardins B, Gorman JH, et al. Increased wall stress of saccular versus fusiform aneurysms of the descending thoracic aorta. Ann Vasc Surg. 2011;25:1129-37.

3. Shang EK, Nathan DP, Sprinkle SR, Fairman RM, Bavaria JE, Gorman RC, et al. Impact of wall thickness and saccular geometry on the computational wall stress of descending thoracic aortic aneurysms. Circulation. 2013;128:S157-62.

4. Shang EK, Nathan DP, Boonn WW, Lys-Dobradin IA, Fairman RM, Woo EY, et al. A modern experience with saccular aortic aneurysms. J Vasc Surg. 2013; $57: 84-8$.
5. Hope MD, Hope TA, Crook SE, Ordovas KG, Urbania TH, Alley MT, et al. 4D flow CMR in assessment of valve-related ascending aortic disease. JACC Cardiovasc Imaging. 2011;4:781-7.

6. Francois CJ, Markl M, Schiebler ML, Niespodzany E, Landgraf BR, Schlensak C, et al. Four-dimensional, flow-sensitive magnetic resonance imaging of blood flow patterns in thoracic aortic dissections. J Thorac Cardiovasc Surg. 2013;145:1359-66.

7. Oechtering TH, Hons CF, Sieren M, Hunold P, Hennemuth A, Huellebrand M, et al. Time-resolved 3-dimensional magnetic resonance phase contrast imaging (4D Flow MRI) analysis of hemodynamics in valve-sparing aortic root repair with an anatomically shaped sinus prosthesis. J Thorac Cardiovasc Surg. 2016;152:418-27.e411.

8. Boussel L, Rayz V, McCulloch C, Martin A, Acevedo-Bolton G, Lawton M, et al. Aneurysm growth occurs at region of low wall shear stress: patient-specific correlation of hemodynamics and growth in a longitudinal study. Stroke. 2008;39: 2997-3002.

9. Jing L, Fan J, Wang Y, Li H, Wang S, Yang X, et al. Morphologic and hemodynamic analysis in the patients with multiple intracranial aneurysms: ruptured versus unruptured. PLoS One. 2015;10:e132494.

10. Lu G, Huang L, Zhang XL, Wang SZ, Hong Y, Hu Z, et al. Influence of hemodynamic factors on rupture of intracranial aneurysms: patient-specific 3D mirror aneurysms model computational fluid dynamics simulation. AJNR Am J Neuroradiol. 2011;32:1255-61.

11. Chisci E, Alamanni N, Iacoponi F, Michelagnoli S, Procacci T, Colombo G, et al. Grading abdominal aortic aneurysm rupture risk. J Cardiovasc Surg (Torino). 2013;57:43S.

12. Burk J, Blanke P, Stankovic Z, Barker A, Russe M, Geiger J, et al. Evaluation of 3D blood flow patterns and wall shear stress in the normal and dilated thoracic aorta using flow-sensitive 4D CMR. J Cardiovasc Magn Reson. 2012;14:84.

13. Callaghan FM, Karkouri J, Broadhouse K, Evin M, Fletcher DF, Grieve SM. Thoracic aortic aneurysm: 4D flow MRI and computational fluid dynamics model. Comput Methods Biomech Biomed Engin. 2015;18(suppl 1): 1894-5.

14. Taylor BV, Kalman PG. Saccular aortic aneurysms. Ann Vasc Surg. 1999;13: $555-9$.

15. Isoda H, Ohkura Y, Kosugi T, Hirano M, Alley MT, Bammer R, et al. Comparison of hemodynamics of intracranial aneurysms between MR fluid dynamics using 3D cine phase-contrast MRI and MR-based computational fluid dynamics. Neuroradiology. 2010;52:913-20.

16. He $\mathrm{X}, \mathrm{Ku} \mathrm{DN}$. Pulsatile flow in the human left coronary artery bifurcation: average conditions. J Biomech Eng. 1996;118:74-82.

17. Long Y, Yu H, Zhuo Z, Zhang Y, Wang Y, Yang X, et al. A geometric scaling model for assessing the impact of aneurysm size ratio on hemodynamic characteristics. Biomed Eng Online. 2014;13:17.

18. Long Y, Zhong J, Yu H, Zhang Y, Wang Y, Yang X, et al. A scaling aneurysm model-based approach to assessing the role of flow pattern and energy loss in aneurysm rupture prediction. J Transl Med. 2015;13:311.

19. Malek AM, Alper SL, Izumo S. Hemodynamic shear stress and its role in atherosclerosis. JAMA. 1999;282:2035-42.

20. Walpola PL, Gotlieb AI, Langille BL. Monocyte adhesion and changes in endothelial cell number, morphology, and F-actin distribution elicited by low shear stress in vivo. Am J Pathol. 1993;142:1392-400.

21. Boyd AJ, Kuhn DC, Lozowy RJ, Kulbisky GP. Low wall shear stress predominates at sites of abdominal aortic aneurysm rupture. J Vasc Surg. 2016;63:1613-9.

22. Motoki T, Kurobe H, Hirata Y, Nakayama T, Kinoshita H, Rocco KA, et al. PPAR-gamma agonist attenuates inflammation in aortic aneurysm patients. Gen Thorac Cardiovasc Surg. 2015;63:565-71.

23. Cheng C, Tempel D, van Haperen R, van der Baan A, Grosveld F, Daemen MJ, et al. Atherosclerotic lesion size and vulnerability are determined by patterns of fluid shear stress. Circulation. 2006;113:2744-53.

Key Words: thoracic aorta aneurysm, saccular aneurysm, 4D-Flow, MRI, hemodynamics 


\section{APPENDIX 1.}

WSS, a friction force of the blood flow on the vessel wall, was defined as follows.

$$
\operatorname{WSS}\left(\mathrm{Pa} / \mathrm{m}^{2}\right)=\frac{4 \mu Q}{\pi r^{3}}
$$

( $\mu=$ fluid viscosity, $Q=$ flow volume, $r=$ radius of vessel)

Viscosity of blood was assumed to be $0.00384 \mathrm{~Pa} / \mathrm{s}$.

The time-averaged WSS is defined as follows.

$$
1 / \mathrm{T} \int_{0}^{\mathrm{T}}\left|w s s_{0}^{t}\right| d t
$$

OSI, mean fluctuation of WSS vectors during 1 cardiac cycle, is defined as follows.

$$
0.5\left[1-\left|\int_{0}^{\mathrm{T}} w s s_{0}^{t} d t\right| / \int_{0}^{\mathrm{T}}\left|w s s_{0}^{t}\right| d t\right]
$$

\begin{tabular}{|c|c|c|c|c|c|c|c|}
\hline & $\begin{array}{c}\text { Group Y, } \\
\mathbf{n}=\mathbf{8}\end{array}$ & $P$ value (vs C) & $\begin{array}{c}\text { Group C, } \\
\mathbf{n}=\mathbf{8}\end{array}$ & $P$ value (vs A) & $\begin{array}{c}\text { Group A, } \\
\mathbf{n}=16\end{array}$ & $P$ value (vs Y) & $P$ value (3 groups) \\
\hline \multicolumn{8}{|l|}{ Ascending aorta } \\
\hline Diameter, mm & $26.33 \pm 1.21$ & .002 & $32.29 \pm 2.96$ & .470 & $34.04 \pm 3.63$ & $<.001$ & $<.001$ \\
\hline Average WSS & $0.44 \pm 0.04$ & .001 & $0.34 \pm 0.04$ & .120 & $0.29 \pm 0.05$ & $<.001$ & $<.001$ \\
\hline Peak WSS & $1.54 \pm 0.27$ & $<.001$ & $0.77 \pm 0.14$ & .270 & $0.60 \pm 0.19$ & $<.001$ & $<.001$ \\
\hline OSI & $0.15 \pm 0.02$ & .034 & $0.19 \pm 0.02$ & .079 & $0.16 \pm 0.04$ & 1.000 & .010 \\
\hline \multicolumn{8}{|l|}{ Aortic arch } \\
\hline Diameter, mm & $24.23 \pm 1.12$ & $<.001$ & $29.73 \pm 2.21$ & .001 & $32.53 \pm 2.81$ & $<.001$ & $<.001$ \\
\hline Average WSS & $0.49 \pm 0.06$ & $<.001$ & $0.32 \pm 0.03$ & .590 & $0.28 \pm 0.05$ & $<.001$ & $<.001$ \\
\hline Peak WSS & $1.68 \pm 0.33$ & $<.001$ & $0.82 \pm 0.13$ & .290 & $0.62 \pm 0.20$ & $<.001$ & $<.001$ \\
\hline OSI & $0.13 \pm 0.02$ & .319 & $0.16 \pm 0.03$ & 1.000 & $0.14 \pm 0.03$ & .405 & .211 \\
\hline Region of aneurysm & & & & & & \multicolumn{2}{|c|}{$P$ value (vs nonaneurysmal aortic arch) } \\
\hline Average WSS & & & & & $0.195 \pm 0.080$ & .001 & \\
\hline Peak WSS & & & & & $0.290 \pm 0.092$ & $<.001$ & \\
\hline OSI & & & & & $0.094 \pm 0.050$ & .003 & \\
\hline
\end{tabular}

TABLE E1. Diameter, average WSS, peak WSS, OSI, in the ascending aorta, aortic arch, and within the aneurysm

WSS, Wall shear stress; OSI, oscillatory shear index; Group Y, young healthy volunteer; Group C, age-matched control; Group A, patients with an aneurysm. 